Document downloaded from:

http://hdl.handle.net/10251/37359

This paper must be cited as:

Salinas Soler, Y.; Agostini, A.; Pérez Esteve, E.; Martínez Mañez, R.; Sancenón Galarza, F.; Marcos Martínez, MD.; Soto Camino, J.... (2013). Fluorogenic detection of Tetryl and TNT explosives using nanoscopic-capped mesoporous hybrid materials. Journal of Materials Chemistry. 1(11):3561-3564. doi:10.1039/C3TA01438J.

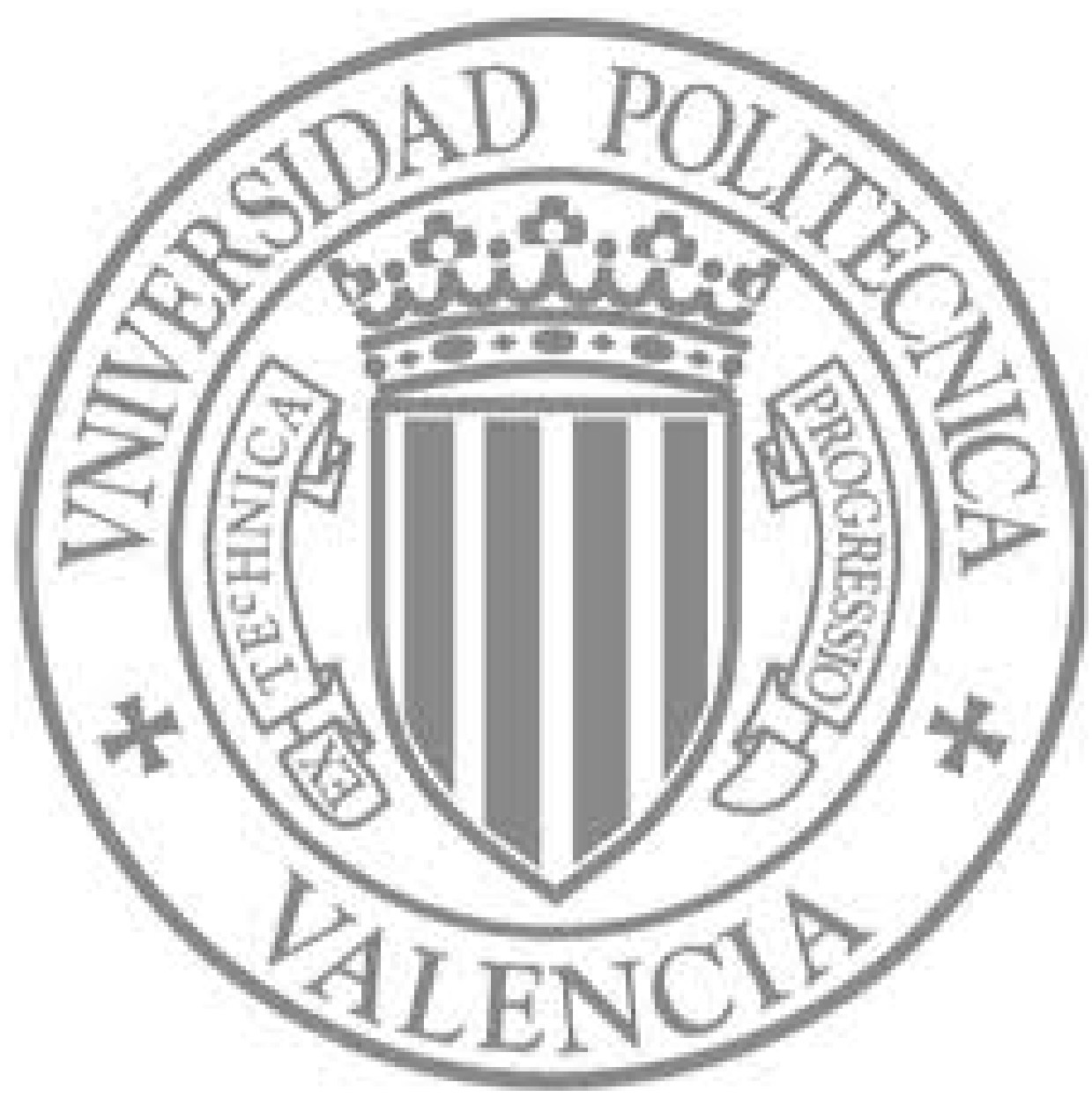

The final publication is available at

http://dx.doi.org/10.1039/C3TA01438J

Copyright Royal Society of Chemistry 


\title{
Fluorogenic detection of Tetryl and TNT explosives using nanoscopic- capped mesoporous hybrid materials
}

\author{
Yolanda Salinas, ${ }^{a, b, c}$ Alessandro Agostini, ${ }^{a, b, c}$ Edgar Pérez-Esteve, ${ }^{a, d}$ Ramón Martínez-Máñez, ${ }^{* a, b, c}$ Félix \\ Sancenón, ${ }^{a, b, c}$ M. Dolores Marcos, ${ }^{a, b, c}$ Juan Soto ${ }^{a, b}$ Ana M. Costero, ${ }^{a, e}$ Salvador Gil ${ }^{a, e}$ Margarita Parra, ${ }^{a, e}$ \\ Pedro Amorós $f$
}

\section{Received (in $X X X, X X X) X$ th $X X X X X X X X X 20 X X$, Accepted Xth $X X X X X X X X X 20 X X$ \\ DOI: 10.1039/b000000x}

A hybrid capped mesoporous material, which was selectively opened in the presence of Tetryl and TNT, has been 10 synthesised and used for the fluorogenic recognition of these nitroaromatic explosives.

Current international public awareness on terrorist attacks using explosives means that the development of quick, reliable methods to detect these chemicals is a particularly interesting issue. ${ }^{1}$ 15 Additionally, given the widespread use of explosive formulations, the analysis of explosives is also of interest in forensic research, landmine detection, and to study environmental problems associated with explosive residues. Among the different chemical explosives, nitroaromatics are perhaps the most commonly used ${ }_{20}$ Current methodologies employed for the detection of nitroaromatic explosives are enzymatic assays, ${ }^{2}$ gas and liquid chromatography, ${ }^{3}$ mass spectrometry, ${ }^{4}$ ion-mobility spectroscopy, ${ }^{5}$ optodes $^{6}$ and electrochemical procedures. ${ }^{7}$ One alternative to these methods, which has recently attracted a great ${ }_{25}$ deal of attention, are optical detection probes based on the design of fluorimetric assays. In most cases, the designed probes are based on the electron-deficient character of nitroaromatic explosives that induces them to form charge-transfer complexes with electron donor molecules (such as polycyclic aromatic 30 hydrocarbons, dansyl derivatives or tetrathiafulvalenes). ${ }^{8}$ Based on this concept, fluorescent polymers, ${ }^{9}$ fluorescent silica-based materials ${ }^{10}$ and single fluorophores ${ }^{11}$ such as probes for the detection of nitroaromatic explosives have been explored.

${ }^{a}$ Centro de Reconocimiento Molecular y Desarrollo Tecnológico (IDM), Unidad Mixta Universidad Politécnica de Valencia-Universidad de Valencia, Spain

${ }^{b}$ Departamento de Química, Universidad Politécnica de Valencia, Camino de Vera s/n, 46022, Valencia, Spain. E-mail: rmaez@qim.upv.es

${ }^{c}$ CIBER de Bioingeniería, Biomateriales y Nanomedicina (CIBER-BBN).

${ }^{d}$ Grupo de Investigación e Innovación Alimentaria, Departamento de Tecnología de Alimentos, Universitat Politècnica de València, Camino de Vera s/n., 46022 Valencia, Spain.

${ }^{e}$ Departamento de Química Orgánica, Facultad de Ciencias Químicas, Universitat de Valencia, 46100 Burjassot, Valencia, Spain. E-mail: ana.costero@uv.es

${ }^{f}$ Institut de Ciència dels Materials (ICMUV), Universitat de Valencia, P.O. Box 2085, E-46071, Valencia, Spain.

$\dagger$ Electronic Supplementary Information (ESI) available: for experimental details, synthesis, characterisation and sensing procedures. See http://dx.doi.org/10.1039/b000000x/
However in many cases, one common drawback in these systems 35 is that fluorescence quenching, involving an electron transfer process between the corresponding excited fluorophore and electron acceptor nitroaromatic explosive molecules, is observed. Nevertheless, reagents which exhibit a turn-on behaviour, rather than the turn-off response, are preferred for designing efficient 40 probes for practical applications. Moreover in the literature, reports on fluorescence-based probes with a turn-on response for nitroaromatic explosives are very limited. ${ }^{12}$

By making full use of the good properties of electron-rich fluorophores, such as pyrene to form complexes with 45 nitroaromatic explosives, and by attempting to design probes showing enhanced fluorescence upon interaction with target nitroaromatics at the same time, we report herein the use of a capped mesoporous material that is selectively uncapped in the presence of nitroaromatic explosives, resulting in the delivery of so a selected fluorophore. Despite some examples reported by us, the use of capped materials to selectively detect target analytes is a barely explored approach. ${ }^{13}$

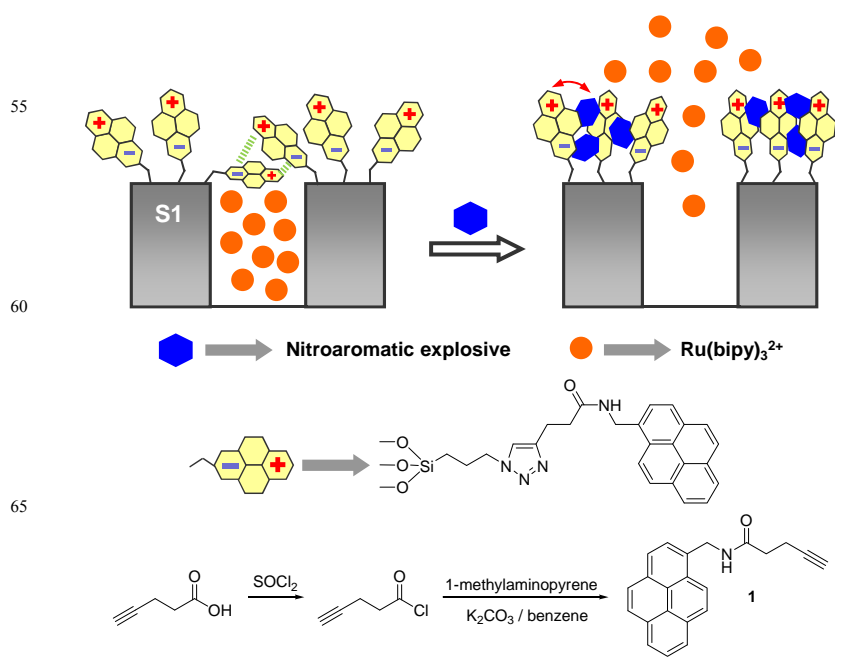

70

Scheme 1. Schematic representation of the sensing mechanism of solid S1 in the presence of explosives and synthesis of pyrene derivative $\mathbf{1}$.

An illustration of the signalling paradigm is shown in Scheme 75 1. As an inorganic support, a mesoporous MCM-41 phase was 
selected given its high loading capacity and easy surface functionalisation. The mesoporous support was first loaded with the dye tris(2,2'-bipyridyl)ruthenium(II) chloride $\left(\left[\mathrm{Ru}(\text { bipy })_{3}\right] \mathrm{Cl}_{2}\right)$ and was then functionalised with 35 (azidopropyl)triethoxysilane. Finally, the support was capped, through a click chemistry reaction, using pyrene derivative $\mathbf{1}$ (see Supporting Information for details). The presence of a dense network of bulky pyrene moieties around the pores was expected to inhibit the delivery of the dye. The signalling protocol relies on

10 pyrene-nitroaromatic complexes formation, which would push apart the bulky pyrene from the pore outlets (to unlock the gate) and the delivery of the dye.

The MCM-41 mesoporous support was prepared by using tetraethyl orthosilicate (TEOS) as a hydrolytic inorganic 15 precursor and the surfactant hexadecyl-trimethylammonium bromide (CTABr) as a porogen species. ${ }^{14}$ After the surfactant was removed by calcination, the MCM-41 solid was obtained. The MCM-41 structure of the starting material was confirmed by XRay diffraction and transmission electron microscopy (TEM), see 20 Supporting Information. The $\mathrm{N}_{2}$ adsorption-desorption isotherms of the prepared phase show a typical type IV-curve (see Figure 1) with a specific surface area of $1415 \mathrm{~m}^{2} \mathrm{~g}^{-1}$, a narrow pore size distribution and an average pore diameter of $2.3 \mathrm{~nm}$. The inorganic support was then loaded with $\left[\mathrm{Ru}(\text { bipy })_{3}\right]^{2+}$ as a suitable 25 fluorophore and the outer surface was functionalised with 3(azidopropyl)triethoxisylane groups. In a second step, pyrene derivative 1 was attached to the solid support by a copper (I)catalysed Huisgen azide/alkyne 1,3-dipolar cycloaddition "click" reaction. ${ }^{15}$ This led to the formation of a 1,2,3-triazole

30 heterocycle and yielded the final hybrid material S1. The solid was filtered, intensively washed with acetonitrile and dried overnight at $36^{\circ} \mathrm{C}$.

35

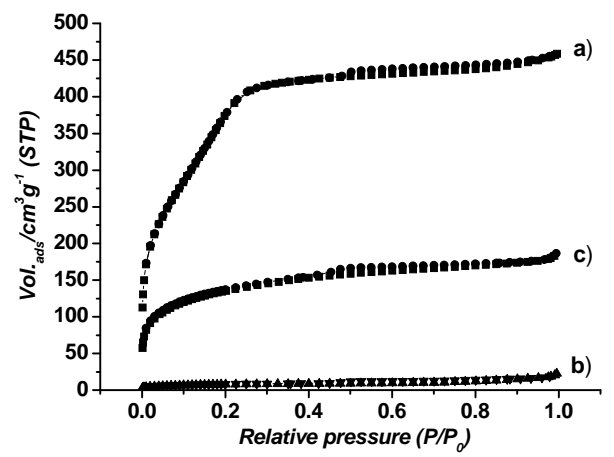

45

Figure 1. $\mathrm{N}_{2}$ adsorption-desorption isotherms of (a) calcined MCM-41 (b) MCM-41 loaded with $\left[\mathrm{Ru}(\text { bipy })_{3}\right]^{2+}$ and functionalized with azide and (c) solid S1.

50 S1 displays the expected features of the MCM-41 phase, indicating that the mesoporous structure was preserved throughout the filling process with the dye and the anchoring of the pyrene derivative at the pore outlets (see Supporting Information). Moreover, TEM images of the MCM-41 support 55 and of the final hybrid material S1 showed the typical porosity associated with the inorganic support (see Supporting Information). The $\mathrm{N}_{2}$ adsorption-desorption isotherm of S1 (see Figure 1) was typical of gated mesoporous systems with partially filled mesopores, and a significant decrease in the $\mathrm{N}_{2}$ volume 60 adsorbed was observed when compared with the starting MCM41 material. The organic content in S1 was determined by thermogravimetric analysis; this solid contained $0.061 \mathrm{mmol}$ of the ruthenium complex $/ \mathrm{g} \mathrm{SiO}_{2}$ and $0.104 \mathrm{mmol}$ of pyrene derivative/g $\mathrm{SiO}_{2}$ (see Supporting Information). DLS (Dynamic

${ }_{65}$ Light Scattering) studies in acetonitrile showed microparticles with a mean diameter of $30.2 \mu \mathrm{m}$ (see Supporting Information).

The studies into the explosive-induced uncapping paradigm were carried out in acetonitrile. In this solvent, a remarkably rather tight pore closure was observed and, at the same time, both 70 the ruthenium dye and the selected explosives showed suitable solubility (see Supporting Information). In a typical assay, $4 \mathrm{mg}$ of S1 were suspended in $10 \mathrm{~mL}$ of acetonitrile in the presence of different explosives at $25^{\circ} \mathrm{C}$. Delivery of the dye to the bulk solution was easily detected by monitoring the spin allowed $\mathrm{d}-\pi$ 75 metal-to-ligand charge transfer (MLCT) transition band of the $\left[\mathrm{Ru}(\text { bipy })_{3}\right]^{2+}$ complex centred at $453 \mathrm{~nm}$, or through its luminescence centred at $625 \mathrm{~nm}\left(\lambda_{\mathrm{ex}}=453 \mathrm{~nm}\right) .{ }^{16}$ Figure 2 shows the release kinetics of the ruthenium dye from $\mathbf{S 1}$ in the presence of explosives Tetryl and TNT at $2.0 \times 10^{-3} \mathrm{~mol} \mathrm{dm}^{-3}$. In the 80 presence of both explosives, a clear dye delivery took place, reaching a maximum release at ca. 15 minutes.

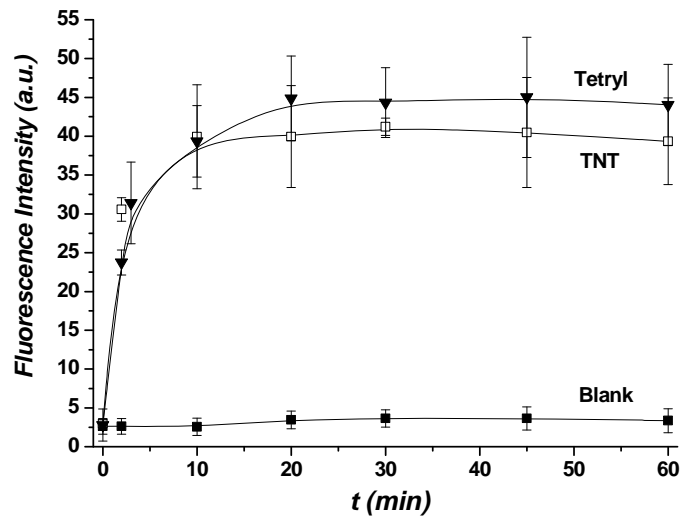

Figure 2. Kinetics of the release of the $\left[\mathrm{Ru}(\text { bipy })_{3}\right]^{2+}$ dye from solid S1 (at $25^{\circ} \mathrm{C}$ ) in the absence (blank) and the presence of nitroaromatic explosives $\left(2.0 \times 10^{-3} \mathrm{~mol} \mathrm{dm}^{-3}\right)$ in acetonitrile $\left(\lambda_{\mathrm{ex}}=453 \mathrm{~nm}, \lambda_{\mathrm{em}}=625 \mathrm{~nm}\right)$

100 In order to prove the proposed sensing mechanism (see Scheme 1), the interaction of Tetryl and TNT with the anchored pyrene groups was explored by the former's ability to quench the latter's excited state. The acetonitrile suspensions of $\mathbf{S 1}\left(\right.$ at $25^{\circ} \mathrm{C}$ ) displayed the typical structured emission of the pyrene monomer 105 in the 380-430 $\mathrm{nm}$ range (see Supporting Information). Addition of Tetryl and TNT to the acetonitrile suspensions of S1 induced a quenching of the monomer emission $(89 \%$ and $93 \%$ of the quenching for TNT and Tetryl, respectively, for an explosive concentration of $2.0 \times 10^{-3} \mathrm{~mol} \mathrm{dm}^{-3}$ ), indicative of the formation 110 of complexes between the electron donor pyrene ring and the electron-withdrawing nitroaromatic explosives. In addition, these Tetryl- and TNT-pyrene interactions were responsible for the dye delivery and the enhanced turn-on luminescence signal observed.

Besides, both facts, i.e., the appearance of the $\left[\mathrm{Ru}(\text { bipy })_{3}\right]^{2+}$ 115 luminescence and the pyrene emission quenching upon addition of TNT and Tetryl, would allow the ratiometric detection of both 
nitroaromatic explosives (see Supporting Information).

In the absence of the nitroaromatic explosives the bulky pyrene inhibited dye delivery due to a partial pore blocking. This blocking may probably arise from strong dipole-dipole 5 interactions between the anchored pyrene derivatives. In fact the gating molecule (containing an electron donor pyrene moiety and a 1,2,3-triazole heterocycle group with an electron acceptor character) has a strong dipole moment of ca. $3.2 \mathrm{D}$ (see Supporting Information). Bearing in mind this value and the use 10 of acetonitrile as solvent, dipole-dipole interactions between the anchored molecules would impose some rigidity and would induce the interaction between gating molecules in the pore voids. This may be favored by the relatively short distance between anchored pyrenes groups (ca. $0.78 \mathrm{~nm}$ ) and the presence 15 of about ten fluorophores surrounding each pore as average (see Supporting Information for details). As the experimental results suggested, Tetryl and TNT interacted with the pyrene group disrupting pyrene-pyrene interactions and inducing dye delivery.

Moreover, in order to discard a simple possible nitroaromatic20 induced displacement of the ruthenium complex additional experiments were carried out. In particular, the chromogenic response in the presence of Tetryl and TNT of a hybrid solid that contains the ruthenium complex in the pores and azide moieties, in the external surface, was tested. $4 \mathrm{mg}$ of solid were suspended 25 in acetonitrile or in acetonitrile containing Tetryl or TNT. The suspensions were stirred and then filtered. In these experiments the same absorbance and emission intensity in the bulk solution for the solid alone and for the solid in the presence of Tetryl and TNT was obtained indicating that there is no a preferential 30 displacement of the ruthenium complex by the nitroaromatic explosives.

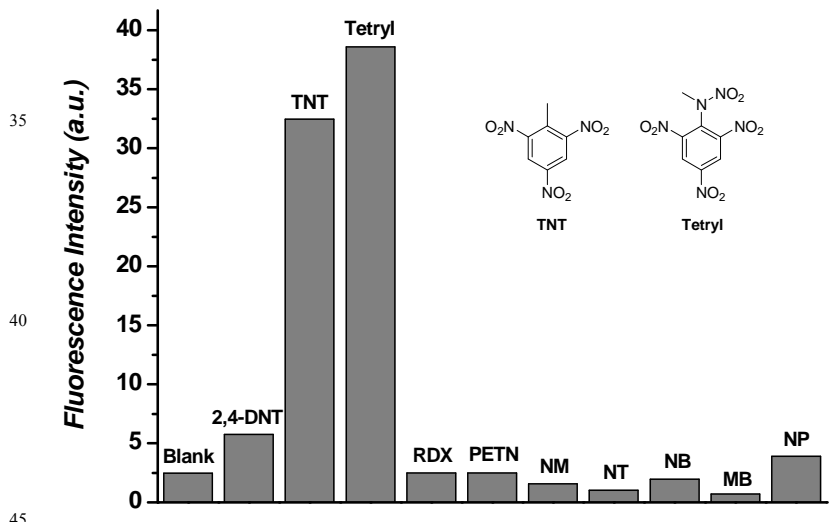

Figure 3. Emission intensity at $625 \mathrm{~nm}$ of acetonitrile suspensions of S1 (at $\left.25^{\circ} \mathrm{C}\right)$ in the presence of different nitroaromatic $\left(2 \times 10^{-3} \mathrm{~mol} \mathrm{dm}^{-3}\right)$ and non-aromatic $\left(2 \times 10^{-3} \mathrm{~mol} \mathrm{dm}^{-3}\right)$ derivatives.

50 Further studies were carried out and titration curves in the presence of Tetryl and TNT were obtained. The increasing intensity in the emission band of the $\left[\mathrm{Ru}(\text { bipy })_{3}\right]^{2+}$ dye was found to be proportional to the concentration of the explosives, which is in agreement with the above-described assisted uncapping ${ }_{55}$ protocol. A simple fluorogenic titration allowed us to calculate detection limits of 1.4 and $11.4 \mathrm{ppm}$ for the detection of Tetryl and TNT, respectively. The detection limits were also calculated from the nitroaromatic-induced quenching of the pyrene emission. Using this procedure, detection limits of 8.6 and 9.1 ${ }_{60} \mathrm{ppm}$ for Tetryl and TNT were calculated.

Finally, in order to check the selectivity of S1 to Tetryl and TNT, the response of the hybrid material in the presence of other aromatic derivatives (i.e., 2,4-dinitrotoluene (2,4-DNT), $\mathrm{N}$ methylaniline (NM), 2-nitrotoluene (NT) and nitrobenzene (NB)), 65 non-aromatic explosives (i.e., hexahydro-1,3,5-trinitro-1,3,5triazine (RDX) and pentaerythritol tetranitrate (PETN)), methylene blue (MB) and naphthalene (NP) was studied. As seen in Figure 3, only 2,4-DNT was also able to induce a partial release of the ruthenium complex, but to a much lesser extent, 70 whereas no delivery from S1 alone and S1 in the presence of other tested products was noted. The observed selectivity was related with the strong electron acceptor character of TNT (with 3 nitro groups) and Tetryl (4 nitro groups) when compared with the other nitro compounds tested. Addition of TNT and Tetryl 75 induced the formation of charge-transfer complexes with the grafted pyrene allowing dye release.

In summary, a novel approach for the fluorimetric sensing of nitroaromatic explosives using silica gated mesoporous hybrid materials has been reported. The presence of explosives Tetryl 80 and TNT in the acetonitrile suspensions of the hybrid material induced a pore opening with the subsequent release of an entrapped dye. By using this simple procedure, fluorescence in the presence of nitroaromatic explosives was observed. In fact, as far as we know, this is one of the very few examples showing an 85 off-on fluorescence in the presence of nitro explosives. Despite the relatively high limit of detection obtained, in the ppm range, one advantage of our hybrid material, when compared with molecular receptors, is its modular character. In particular, the possible functionalisation of the external surface of mesoporous 90 materials with other gating electron-deficient groups (to enhance or tune selectivity) and the possible use of different fluorophores make this approach appealing for the future development of materials with tailor-made sensing properties showing a turn-on chromo-fluorogenic behaviour for the easy detection of 95 explosives and current efforts into this direction are being carried out in our research group.

\section{Acknowledgments}

${ }_{100}$ Financial support from the Spanish Government (project MAT2012-38429-C04-01) and the Generalitat Valencia (project PROMETEO/2009/016) is gratefully acknowledged. Y.S. is grateful to the Spanish Ministry of Science and Innovation for her grant. A. A. also thanks the Generalitat Valenciana for his 105 Santiago Grisolia fellowship.

\section{References}

1 (a) S. Singh, J. Haz. Mat., 2007, 144, 15. (b) R. Schulte-Ladbeck, M. Vogel, U. Karst, Anal. Bioanal. Chem., 2006, 386, 559.

2 R. G. Smith, N. D’Souza, S. Nicklin, Analyst, 2008, 133, 571.

1103 D. S. Moore, Rev. Sci. Instrum., 2004, 75, 2499.

4 K. Hakansson, R. V. Coorey, R. A. Zubarev, V. L. Talrose P. Hakansson, J. Mass Spectrom., 2000, 35, 337.

5 E. Wallis, T. M. Griffin, N. Popkie, Jr., M. A. Eagan, R. F. McAtee, D.

Vrazel, J. McKinly, Proc. SPIE-Int. Soc.Opt. Eng., 2005, 5795, 54. 1156 M. E. Germain, M. J. Knapp, Chem. Soc. Rev., 2009, 38, 2543. 
7 E. S. Forzani, D. Lu, M. J. Leright, A. D. Aguilar, F. Tsow, R. A. Iglesias, Q. Zhang, J. Lu, J. Li, N. Tao, J. Am. Chem. Soc., 2009, 131, 1390.

8 Y. Salinas, R. Martínez-Máñez, M. D. Marcos, F. Sancenón, A. M. 5 Costero, M. Parra, S. Gil, Chem. Soc. Rev., 2012, 41, 1261.

9 S. W. Thomas III, G. D. Joly, T. M. Swager, Chem. Rev., 2007, 107, 1339.

10 See for example: (a) D. Gao, Z. Wang, B. Liu, L. Ni, M. Wu, Z. Zhang, Anal. Chem., 2008, 80, 8545. (b) S, Zhang, F. Lu, L. Gao, L. 10 Ding, Y. Fang, Langmuir, 2007, 23, 1584.

11 See for example: (a) A. D. Hughes, I. C. Glenn, A. D. Patrick, A. Ellington, E. V. Anslyn, Chem. Eur. J., 2008, 14, 1822. (b) C. Vijayakumar, G. Tobin, W. SchmittM. Kima, M. Takeuchi, Chem. Commun., 2010, 46, 874.

15 12. Y. Salinas, E. Climent, R. Martínez-Máñez, F. Sancenón, M. D. Marcos, J. Soto, A. M. Costero, S. Gil, M. Parra, A. Pérez de Diego, Chem. Commun., 2011, 47, 11885.

13 R. (a) E. Climent, R. Martínez-Mañez, F. Sancenón, M. D. Marcos, J. Soto, A. Maquieira, P. Amorós, Angew. Chem. Int. Ed.., 2010, 49, 7281.

20 (b) E. Climent, M. D. Marcos, R. Martínez-Máñez, F. Sancenón, J. Soto, K. Rurack, P. Amorós, Angew. Chem. Int. Ed., 2009, 48, 8519. (c) E. Climent, A. Bernardos, R. Martínez-Máñez, A. Maquieira, M. D. Marcos, N. Pastor-Navarro, R. Puchades, F. Sancenón, J. Soto, P. Amorós, J. Am. Chem. Soc., 2009, 131, 14075.

2514 S. Cabrera, J. El Haskouri, C. Guillem, J. Latorre, A. Beltrán, D. Beltrán, M. D. Marcos, P. Amorós, Solid State Sci., 2000, 2, 405.

15 H. C. Kolb, M. G. Finn, K. B. Sharpless, Angew. Chem. Int. Ed., 2001, 40, 2004.

16 (a) F. Felix, J. Ferguson, H. U. Gudel, A. Ludi, J. Am. Chem. Soc., 30 1980, 102, 4096. (b) F. E. Lytle, D. M. Hercules, J. Am. Chem. Soc., $1969,91,253$. 